Original article

\title{
Vaginal microbiome diversity and preterm birth: results of a nested case-control study in Peru
}

\author{
Freida Blostein, $M P H^{a}$, Bizu Gelaye, PhD ${ }^{b}$, Sixto E. Sanchez, MD, MPH ${ }^{c}$, \\ Michelle A. Williams, PhD ${ }^{b}$, Betsy Foxman, PhD ${ }^{\text {a, * }}$ \\ a University of Michigan Thomas Francis School of Public Health, Ann Arbor, MI \\ ${ }^{\mathrm{b}}$ Harvard T. H. Chan School of Public Health, Boston, MA \\ ${ }^{\mathrm{c}}$ Universidad de San Martín de Porres, Facultad de Medicina, Lima, Perú
}

\section{A R T I C L E I N F O}

\section{Article history:}

Received 26 February 2019

Accepted 26 November 2019

Available online 5 December 2019

\section{Keywords:}

Premature birth

Microbiota

Host-microbial interactions

Molecular epidemiology

\begin{abstract}
A B S T R A C T
Purpose: Preterm birth (PTB) is a major cause of neonatal mortality. The vaginal microbiome is associated with PTB, but results vary across racial/ethnic populations. Some evidence suggests gestational age affects this association. We investigated these associations in a novel population, conducting a post hoc analysis assessing if associations differed between women swabbed at different gestational ages. Methods: We compared vaginal microbiomes from women with PTB $(n=25)$ to a random sample of women with term births $(n=100)$ among participants in the Pregnancy Outcomes, Maternal and Infant Study, conducted in Lima, Peru. Using DADA2, we identified taxa from 16S DNA sequencing and used Dirichlet multinomial mixture models to group into community state types (CSTs).

Results: If gestational age at sampling was not considered, no CST (diverse, Lactobacillus-dominated or Lactobacillus iners-dominated), was associated with PTB. Among women sampled before 12 weeks' gestation, women with Lactobacillus-dominated CSTs were less likely to have a PTB than those with a diverse CST. Among those swabbed between 12 and 16 weeks' gestation, the reverse was true.

Conclusions: Our study supports previous literature suggesting that what constitutes a healthy vaginal microbiome varies by race/ethnicity. Longitudinal studies are necessary to disentangle effects of vaginal microbiome differences over gestation.
\end{abstract}

(๑) 2019 Elsevier Inc. All rights reserved.

\section{Introduction}

Preterm birth (PTB) is a major cause of neonatal mortality worldwide [1-3]. Infants born preterm are more likely to suffer from respiratory distress syndrome, necrotizing enterocolitis, and intraventricular hemorrhage and developmental delays [2,4,5]. Twenty-five to thirty percent of preterm births are attributed to intrauterine infection and subsequent immune response [4,6,7]. The most common pathway to intrauterine infection is ascent from the vagina and cervix [4]; bacterial vaginosis (BV) is associated with

Conflicts of Interest statement: The authors have no conflicts of interest to disclose.

Paper Presentation Information: An earlier version of the work was presented at the 2018 American Epidemiology Society Meeting, Baltimore, Maryland.

* Corresponding author. University of Michigan Thomas Francis School of Public Health, M5108 SPH II, 1415 Washington Heights, Ann Arbor, MI, 48109. Tel.: +(734)764-5487; fax: +(734)-764-3192.

E-mail address: bfoxman@umich.edu (B. Foxman).
1.5- to 3-fold increases risk for PTB [4]. However, interventions targeting BV have been unsuccessful [6,8].

Next-generation sequencing technologies make it possible to characterize complex microbial communities associated with PTB [9-12]. However, findings from studies deploying these novel methods have been inconsistent, due in part to differences in study design, populations studied, and bioinformatics and statistical analytical techniques [2,13-15]. Nonetheless, available evidence suggests that the vaginal microbiome among pregnant women is characterized by increased community stability and decreased community diversity when compared with their nonpregnant counterparts [2,16]. For example, Aagaard et al. reported that the Shannon diversity (a measure of species diversity in a community) of the vaginal microbiome decreased with increasing gestational age among pregnant women [16]. This observation was corroborated by Stout et al. using a cohort mostly of African American women. However, following a comparison of a predominantly Caucasian cohort to a predominantly African American cohort, Callahan et al. concluded that PTB microbiota associations are population dependent [13]. Specifically, Lactobacillus crispatus and 
all Lactobacillus species occurred less frequently in the American American cohort, although in both cohorts there was apparent exclusion of Gardnerella vaginalis by L. crispatus.

The patterns of microbiota changes during pregnancy also may be predictive of PTB. Stout et al. showed that women with subsequent PTB had a significantly greater decrease in vaginal microbiome diversity, richness, and evenness between the first and second trimester compared to those with term deliveries-by the third trimester, there were no differences in the vaginal microbiome by term status [17]. Fettweis et al. found that among women with PTB, taxa associated with BV tended to decrease in abundance throughout pregnancy, whereas L. crispatus increased. Fettweis et al. also noted that race affected changes over time in the vaginal microbiome during pregnancy, with Caucasian women exhibiting more stable microbiomes, although $G$. vaginalis increased among Caucasian women with PTB [18]. On balance, available evidence suggests that gestational age-specific changes in the vaginal microbiome may be predictive of term status, but these findings vary by race/ethnicity. Whether these alterations are risk factors or risk markers of PTB remains uncertain.

Given the available evidence, we conducted a pilot case-control study, nested within a well-characterized prospective cohort of pregnant Peruvian women, to describe the association of maternal early-pregnancy vaginal microbiome signatures, determined using 16S rRNA gene sequence-based methods, with PTB. Our study adds to the growing literature suggesting modifications of the association between changes in the vaginal microbiota during pregnancy and risk of PTB by race/ethnicity.

\section{Materials and methods}

\section{Study population}

The sample for the present study was drawn from participants enrolled in the Pregnancy Outcomes, Maternal and Infant Study (PrOMIS) cohort who provided vaginal swabs $(n=785)$ between October 2013 and May 2014. Details of the PrOMIS cohort have been described previously [19]. Briefly, the study population consists of women attending prenatal care clinics at the Instituto Nacional Materno Perinatal in Lima, Peru. Women who initiated prenatal care before 16 weeks' gestation were eligible; if they were younger than 18 years of age, did not speak and read Spanish, or had completed more than 16 weeks' gestation, they were excluded. Enrolled consenting participants were interviewed by trained research personnel using a structured questionnaire to elicit information regarding maternal sociodemographic, lifestyle characteristics, and medical and reproductive histories. All participants provided written informed consent. The institutional review boards of the Instituto Nacional Materno Perinatal, Lima, Peru, and the Harvard School of Public Health Office of Human Research Administration, Boston, MA, approved all procedures used in this study.

Of the 785 selected participants, 35 women spontaneously delivered or had premature rupture of membranes and delivered at less than 37 weeks of gestation. After excluding women with multiple pregnancies, stillbirth, and medical complications of pregnancy including pre-eclampsia, a total of 25 PTB cases (gestational age at delivery $<37$ completed weeks) remained for microbiome analyses. A total of 529 women had term delivery, single live birth, and no medical complications (i.e., pre-eclampsia and no placental abruption). Randomly sampled 100 women who delivered at term ( $\geq 37$ weeks of gestation; mean $\pm S D=39.0 \pm 1.0)$ were selected as term controls (ratio of $4: 1$ ). The 100 participants selected for this analysis did not differ when compared with all participants in the PrOMIS cohort.

\section{Sample collection}

Maternal vaginal swabs were self-collected using (i) dry Dacron sterile polyester (Starplex Scientific Starswab II Collection and Transport Systems) and (ii) ESwab (Copan Liquid Amies Elution Swab Collection and Transport System) swabs at 9 weeks of gestation, on average. The ESwab swabs were placed in the ESwab tube containing $1 \mathrm{~mL}$ of modified Liquid Amies solution. The swabs were immediately stored on ice and transported to the laboratory for storage at $-80^{\circ} \mathrm{C}$ before processing. The second swab was used to create a vaginal smear on the glass slide for BV analysis.

\section{Definition of preterm birth}

We defined PTB according to the American College of Obstetricians and Gynecologists guidelines [20]. Gestational age was determined using the last menstrual period and confirmed by ultrasound examination, conducted before 20 weeks of gestation. Using information collected from maternal medical records, we categorized PTB cases according to the three pathophysiological groups previously described (i.e., spontaneous PTB, preterm premature rupture of membranes, and medically induced PTB) [21,22]. Women who delivered before 37 completed weeks of gestation as a result of medical intervention were not eligible for this study.

\section{Sequencing}

The 16S rRNA gene V4 variable region PCR primers 515/806 with barcode on the forward primer were used in a 30-cycle PCR using the HotStarTaq Plus Master Mix Kit (Qiagen) under the following conditions: $94^{\circ} \mathrm{C}$ for 3 minutes, followed by 28 cycles of $94^{\circ} \mathrm{C}$ for 30 seconds, $53^{\circ} \mathrm{C}$ for 40 seconds and $72^{\circ} \mathrm{C}$ for 1 minute, after which a final elongation step at $72^{\circ} \mathrm{C}$ for 5 minutes was performed. After amplification, PCR products were checked in $2 \%$ agarose gel to determine the success of amplification and the relative intensity of bands. Multiple samples were pooled together (e.g., 100 samples) in equal proportions based on their molecular weight and DNA concentrations. Pooled samples were purified using calibrated Ampure XP beads. Then the pooled and purified PCR products were used to prepare DNA libraries by following Illumina TruSeq DNA library preparation protocol. Sequencing was performed at MR DNA (www.mrdnalab.com, Shallowater, TX) on a MiSeq following the manufacturer's guidelines. Reagent controls using certified DNAfree water were run through library preparation and PCR and did not generate libraries. For quality control, samples submitted for sequencing included a random sample of technical replicates. All library preparation and sequencing were carried out at MR DNA.

\section{Bioinformatics}

Reads were demultiplexed using idemp and then trimmed of adapters, quality-trimmed, and quality-filtered using DADA2. Of the 8,811,003 total reads, 8,320,983 passed quality filtering. Error rates were learned on a subset of the samples and used to infer sequence variants separately for each run, then paired ends were merged. Runs were then merged, chimeras were removed, and taxonomy was assigned using the Silva v132 database [23] (Supplemental Methods). Technical replicates were visually examined for differences (Supplemental Fig. 2). For a single sample sequenced in triplicate, one replicate did not match the other two replicates and was excluded from further analysis. All other replicates were similar to each other and counts from replicates were summed. We removed any amplicon sequence variants (ASVs) that were not bacterial and collapsed ASVs assigned to the same species together. We conducted diversity analyses on rarefied and 
unrarefied samples. For the community state type (CST) and ALDEX2 analyses, we filtered phyla occurring in $<1$ sample on average and ASVs present at $<00.5 \%$ relative abundance in every sample.

\section{Statistical analysis}

We created Dirichlet multinomial mixture models using R v3.3.2 and the DirichletMultinomial v1.6.0 package [24] to assign all samples to CSTs. We determined the number of CSTs by comparing the Laplace approximation of the negative log models and identifying the point at which an increase in Dirichlet components resulted in minor reductions in model fit. We compared the results of the community state typing to results from a complete linkage hierarchical clustering method using Euclidian distances on the matrix of microbe relative abundances.

We used logistic models to test the association between PTB and CST, controlling for parity and Mestizo ethnicity, as these factors may influence vaginal microbiota and risk of preterm delivery. To test if individual taxa were differentially abundant between term and preterm samples, we used R v3.3.2 and the ALDEX2 package [25]. Logistic models and ALDEX2 analyses were performed on the entire sample as well as separately among those sampled before 12 weeks' gestation ( $n=55$ ) and those sampled at 12 to 16 weeks' gestation $(n=69)$. As sensitivity analyses, we 1$)$ included a parameter modeling dispersion for these models and 2) excluded non-Mestizo individuals because of a lack of non-Mestizo cases sampled at 12 to 16 weeks' gestation (Supplemental Tables 5-8). We calculated measures of multiplicative and additive effect modification (Supplemental Tables 9-11) [26,27]. In addition, we examined key dominating Lactobacillus sp. for patterns of exclusion with Gardnerella sp., as suggested by Callahan et al. [13], stratified by gestational age at sampling.

\section{Results}

\section{Study population}

At an alpha $=0.05$, cases and controls were not statistically significant for any patient demographic or microbial features in the

Table 1

Prevalence of selected sociodemographic, medical, and reproductive health variables by preterm birth status in a sample of Peruvian women

\begin{tabular}{|c|c|c|c|}
\hline Characteristic & $\begin{array}{l}\text { Term } \mathrm{n}=100 \\
\mathrm{~N}(\%) \text { or mean }(\mathrm{SD})\end{array}$ & $\begin{array}{l}\text { Preterm } \mathrm{n}=25 \\
\mathrm{~N}(\%) \text { or mean }(\mathrm{SD})\end{array}$ & $P$-value \\
\hline Community state type & & & .811 \\
\hline Diverse & $50(50.0 \%)$ & $12(48.0 \%)$ & \\
\hline Lactobacillus ASV2 dominated & $26(26.0 \%)$ & $8(32.0 \%)$ & \\
\hline Lactobacillus iners dominated & $24(24.0 \%)$ & $5(20.0 \%)$ & \\
\hline Gestational age at swab & & & .791 \\
\hline$<12$ wk & $54(54.5 \%)$ & $15(60.0 \%)$ & \\
\hline$>$ or $=12-16 \mathrm{wk}$ & $45(45.5 \%)$ & $10(40.0 \%)$ & \\
\hline Shannon diversity & $1.35(0.73)$ & $1.17(0.72)$ & .283 \\
\hline Maternal age & $27.9(6.15)$ & $30.1(7.37)$ & .165 \\
\hline Maternal age (categories) & & & .808 \\
\hline $18-19$ & $6(6.00 \%)$ & $1(4.00 \%)$ & \\
\hline $20-29$ & $57(57.0 \%)$ & $12(48.0 \%)$ & \\
\hline $30-34$ & $19(19.0 \%)$ & $6(24.0 \%)$ & \\
\hline 35 and older & $18(18.0 \%)$ & $6(24.0 \%)$ & \\
\hline Education & & & .075 \\
\hline$>12$ th grade & $47(47.0 \%)$ & $6(24.0 \%)$ & \\
\hline 7 th to 12 th grade & $48(48.0 \%)$ & $18(72.0 \%)$ & \\
\hline$<$ or $=6$ th grade & $5(5.00 \%)$ & $1(4.00 \%)$ & \\
\hline Mestizo & & & .432 \\
\hline No & $26(26.0 \%)$ & $4(16.0 \%)$ & \\
\hline Yes & $74(74.0 \%)$ & $21(84.0 \%)$ & \\
\hline Married & & & .349 \\
\hline No & $23(23.0 \%)$ & $3(12.0 \%)$ & \\
\hline Yes & $77(77.0 \%)$ & $22(88.0 \%)$ & \\
\hline Employment & & & .964 \\
\hline No & $41(41.0 \%)$ & $11(44.0 \%)$ & \\
\hline Yes & $59(59.0 \%)$ & $14(56.0 \%)$ & \\
\hline Trouble paying for basics & & & .754 \\
\hline No & $50(50.0 \%)$ & $11(44.0 \%)$ & \\
\hline Yes & $50(50.0 \%)$ & $14(56.0 \%)$ & \\
\hline Planned pregnancy & & & .349 \\
\hline No & $50(51.0 \%)$ & $16(64.0 \%)$ & \\
\hline Yes & $48(49.0 \%)$ & $9(36.0 \%)$ & \\
\hline Early pregnancy BMI & & & .066 \\
\hline$<18.5$ & $0(0.00 \%)$ & $2(8.00 \%)$ & \\
\hline $18.5-24.9$ & $47(47.0 \%)$ & $11(44.0 \%)$ & \\
\hline $25-29.9$ & $35(35.0 \%)$ & $6(24.0 \%)$ & \\
\hline$>$ or $=30$ & $17(17.0 \%)$ & $5(20.0 \%)$ & \\
\hline Missing & $1(1.00 \%)$ & $1(4.00 \%)$ & \\
\hline Nulliparous & & & 1.000 \\
\hline Parous & $46(46.5 \%)$ & $12(48.0 \%)$ & \\
\hline Nulliparous & $53(53.5 \%)$ & $13(52.0 \%)$ & \\
\hline Bacterial vaginosis (Hay-Ison criteria) & & & .669 \\
\hline I & $15(15.0 \%)$ & $6(24.0 \%)$ & \\
\hline Missing & $3(3.00 \%)$ & $0(0.00 \%)$ & \\
\hline $\mathrm{N}$ & $56(56.0 \%)$ & $14(56.0 \%)$ & \\
\hline VB & $26(26.0 \%)$ & $5(20.0 \%)$ & \\
\hline
\end{tabular}

The second CSTs dominating organism-labeled ASV2-was identified as either L. acidophilus or L. crispatus by a BLAST search. 
unstratified analysis (Table 1 ). Only $24.0 \%$ of cases were educated beyond high school, while $47.0 \%$ of controls were $(P=.075)$. In addition, $8.00 \%$ of cases were underweight in early pregnancy, while no controls were $(P=.066)$.

Mothers sampled at or after 12 to 16 weeks' gestation were slightly younger than mothers sampled before 12 weeks' gestation, with respective mean ages of 26.9 versus $29.6(P=.019)$. No other differences were statistically significant (Supplemental Table 1). Cases sampled at or after 12 weeks' gestation were less likely to be educated beyond high school than controls $(10.0 \%$ vs. $53.3 \%$, $P=.018)$. The same trend existed in those sampled before 12 weeks' gestation, but the difference was not statistically significant $(P=$ .562). All cases among non-Mestizo women had vaginal samples from before 12 weeks' gestation (Supplemental Table 2).

\section{Microbial diversity}

When considered altogether, there were no differences in alpha diversity measures by case status. After stratifying by trimester of microbiome assessment, cases had lower alpha diversity than controls among those sampled at or after 12 weeks' gestation (respective mean Shannon 1.01 vs. $1.49, P=.053$ )-regardless of whether samples were rarefied (Fig. 1). The same relationship held when limiting the sample to Mestizo women (Supplemental Fig. 3). Microbial communities did not cluster together by PTB status or gestational age at sampling on an NMDS plot of Bray-Curtis distances (Supplemental Fig. 4).

\section{Community state types}

Three CSTs emerged from Dirichlet-multinomial modeling. These included 1) a more diverse CST, 2) a CST dominated by a Lactobacillus species (hereafter referred to as Lactobacillus ASV2), and 3) a CST dominated by Lactobacillus iners (Fig. 2). Lactobacillus
ASV2 was identified as either Lactobacillus acidophilus or L. crispatus by a BLAST search.

\section{Microbial characteristics by subject and swab characteristics}

In the unstratified sample, only classification using the Hay-Ison criteria was associated with $\operatorname{CST}(P<.001)$, with more individuals in the diverse CST classified as intermediate or BV (Supplemental Table 3). The same association with Hay-Ison criteria existed in both strata of gestational age at swab. Among those sampled at or after 12-week gestation, more unplanned pregnancies existed in the $L$. iners-dominated CST than in other CSTs $(P=.01)$ (Supplemental Table 4).

\section{Association with PTB}

Overall, no CST was associated with PTB in crude or adjusted logistic models (Table 2). When stratified by timing of microbiome assessment, there was a marginal association $(P<.10)$ with the Lactobacillus-ASV2-dominated CST in the fully adjusted (OR 4.65, 95\% CI: [0.81, 29.96]) model (Table 3). An effect modification analysis suggests the presence of effect-measure modification on the multiplicative (ratio of ORs, Lactobacillus ASV2-dominated vs. diverse CST (95\% CI): $10.41(1.21,101.18) ; P=.04$, L. iners dominated vs. diverse CST ( $95 \% \mathrm{CI}): 9.47(0.86,129.05) ; P=.07)$ but not additive scale (Supplemental Tables 9-11).

\section{Alternative clustering technique}

In dendrograms showing clustering and accompanying heatmaps showing composition, CSTs and the hierarchical clustering technique clustered samples similarly (Fig. 2). Samples collected during 12- to 16-week gestation-but not those collected before 12-week gestation-clustered by CST and preterm status.
A

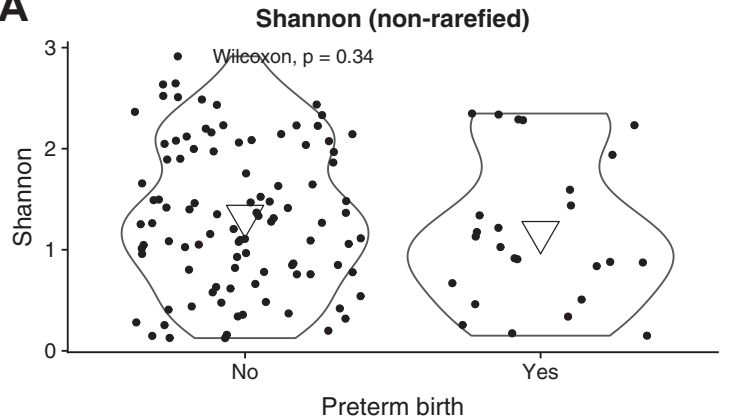

Preterm birth

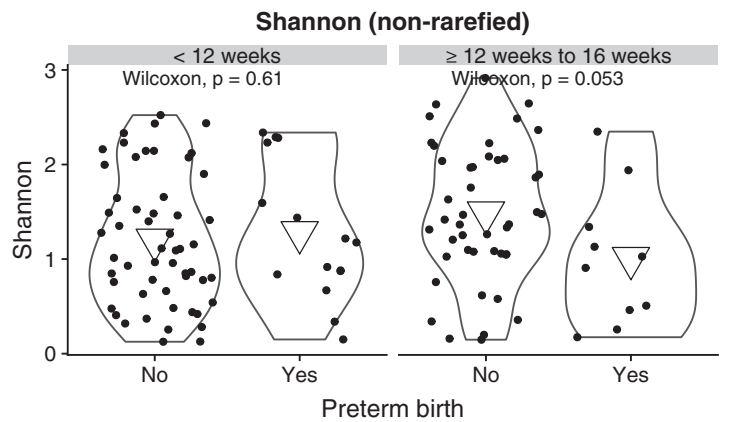

B
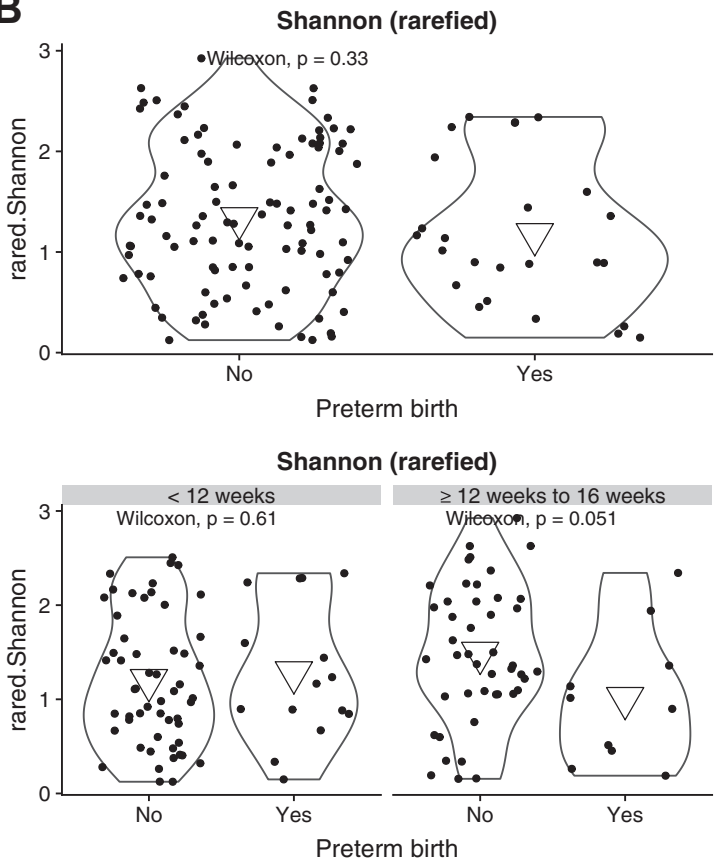

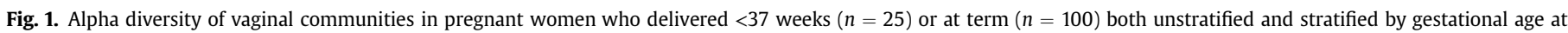

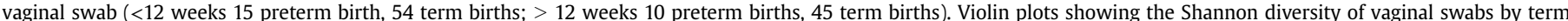

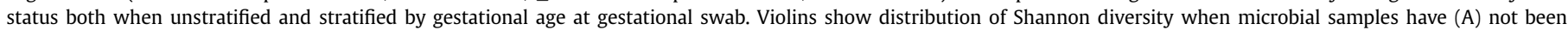
rarefied to even depth and (B) have been rarefied to even depth. Means shown as large, inverted triangles. 
A

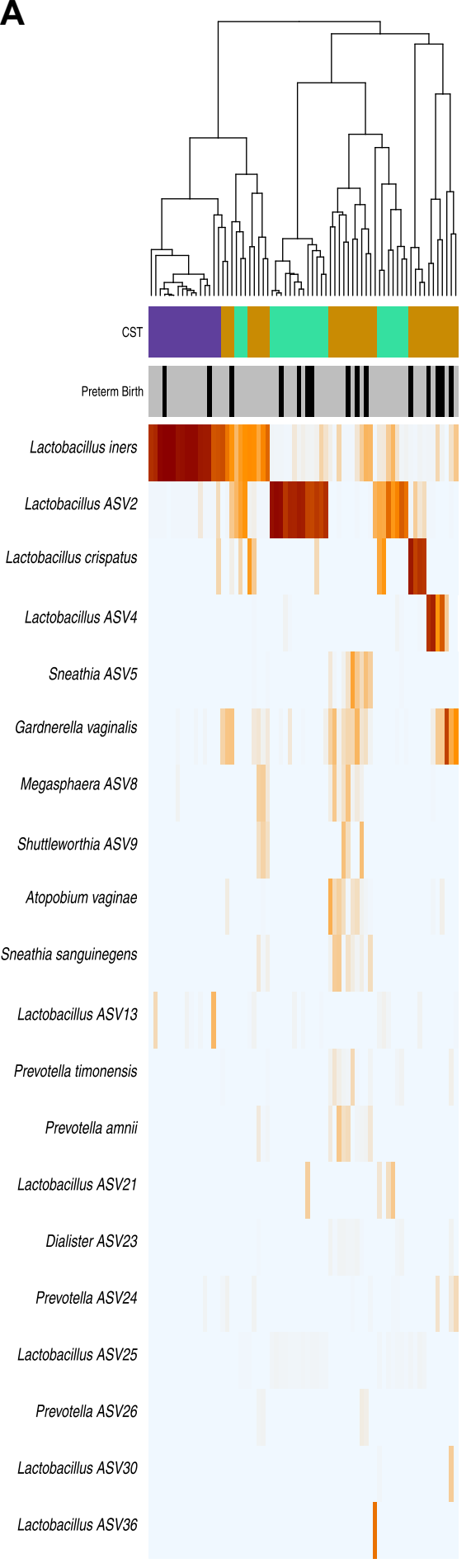

B
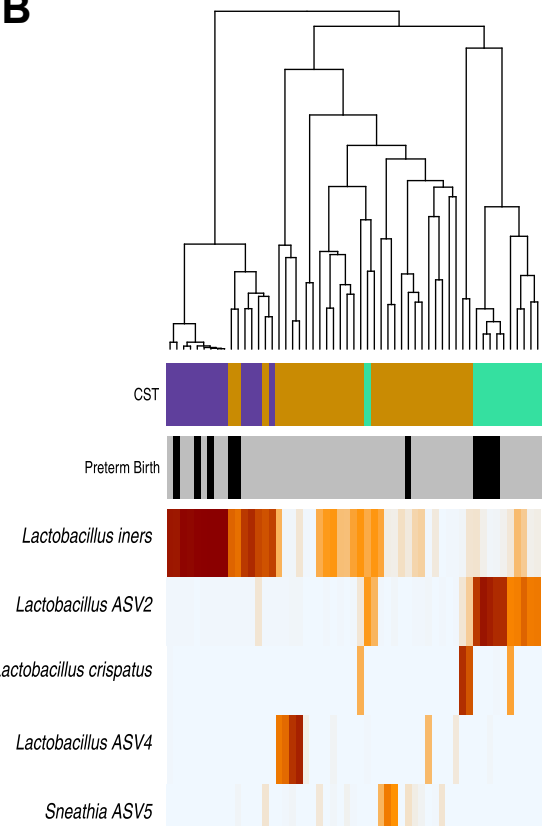

Gardnerella vaginalis

Megasphaera ASV8

Shuttleworthia ASVg

Atopobium vaginae

Sneathia sanguinegens

Lactobacillus ASV13

Prevotella timonensis

Prevotella amnii

Lactobacillus ASV21

Dialister ASV23

Prevotella ASV24

Lactobacillus ASV25

Prevotella ASV26

Lactobacillus ASV30

Lactobacillus ASV 36

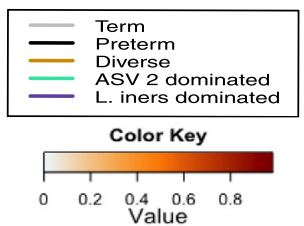

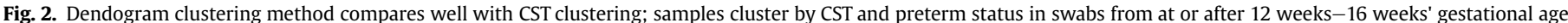

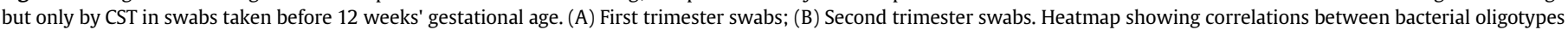

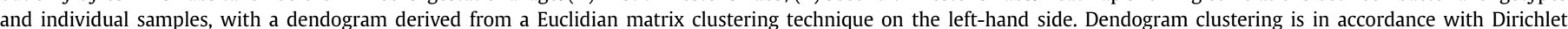

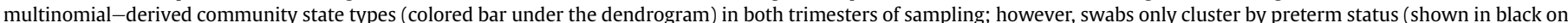
bar directly on the heatmap) in the second trimester swabs. 
Table 2

Results of logistic regression for preterm birth status (odds ratios and 95\% CI) in a sample of Peruvian women

\begin{tabular}{|c|c|c|c|}
\hline Characteristic & $\begin{array}{l}\text { Model } 1 * \text { OR }(95 \% \mathrm{CI})^{5} \\
25 \text { cases } / 100 \text { controls }\end{array}$ & $\begin{array}{l}\text { Model } 2^{\dagger} \text { OR }(95 \% \mathrm{CI})^{5} \\
25 \text { cases/99 controls }\end{array}$ & $\begin{array}{l}\text { Model } 3^{\ddagger} \text { OR }(95 \% \mathrm{CI})^{5} \\
25 \text { cases/99 controls }\end{array}$ \\
\hline Lactobacillus ASV2 $\|$ dominated (reference diverse community) & $1.28(0.45,3.5)$ & $1.25(0.43,3.49)$ & $1.27(0.44,3.56)$ \\
\hline L. iners dominated (reference diverse community) & $0.87(0.25,2.64)$ & $0.85(0.25,2.59)$ & $0.87(0.25,2.68)$ \\
\hline
\end{tabular}

* Unadjusted.

${ }^{\dagger}$ Adjusted for parity status (one individual missing parity status).

Adjusted for parity and Mestizo status (one individual missing parity status).

Profile likelihood-based confidence intervals reported.

\| The second CSTs dominating organism-labeled ASV2-was identified as either L. acidophilus or L. crispatus by a BLAST search.

\section{Joint distribution of key taxa}

We examined the joint distribution of Lactobacillus species with Gardnerella species. Consistent with the analysis by Callahan et al., we observed that Gardnerella species displayed exclusionary patterning with $L$. crispatus and a co-occurring pattern with $L$. iners [13]. In our sample, Lactobacillus ASV2 appeared to have an exclusionary pattern with Gardnerella (Supplemental Fig. 7).

\section{Individual taxa}

No taxa associated with PTB in unstratified or stratified analysis after Benjamini-Hochberg correction for multiple testing. Before correction for multiple testing, two ASVs were associated $(P<.05)$ in the first trimester (Supplemental Figs. 8-9).

\section{Discussion}

We conducted a 16S rRNA gene taxonomic screen characterizing the vaginal microbiome during the first and early second trimester of gestation among 25 PTB cases and 100 term controls sampled from a well characterized cohort of pregnant Peruvian women. We identified three community states: one dominated by L. iners, one by another Lactobacillus species, and one more diverse. Vaginal microbial communities did not associate with PTB overall. The direction of associations between Lactobacillus-dominated communities and PTB differed between women sampled at different gestational ages. The finding that vaginal microbiomes do not associate with PTB is consistent with some results of studies conducted among non-Caucasian women $[2,13]$. Thus, our results support the hypothesis that Lactobacillus-dominated community types are not universal indicators of a healthy pregnant vaginal microbiome in all populations.

The types of vaginal microbial communities in our sample are similar to those reported elsewhere, including communities dominated by Lactobacillus species and more diverse communities. The Peruvian women in our sample have vaginal microbiota more similar to African American and Hispanic US women than to Caucasian or Asian women [28,29].

In the unstratified analysis, we found no association between vaginal communities and PTB. This contradicts previous literature suggesting a protective association between Lactobacillus-dominated vaginal communities and PTB [13-15]. However, studies among non-Caucasian women have reported either weaker or null associations of Lactobacillus-dominated communities with PTB $[2,13,30]$. In a primarily African American cohort, Romero et al. reported no associations of microbial CSTs with PTB [2]. Callahan et al. found no statistically significant differences in relative abundances of Lactobacillus or Gardnerella by term status in a cohort of predominately African American women although they did report statistically significant difference of $L$. crispatus [13]. Among a mixed cohort of both African American and Caucasian women, Fettweis et al. reported term women had higher abundances of $L$. crispatus than preterm women, but when stratified by race, these differences were greater among Caucasian women than among African American women [18]. Vaginal microbiota and PTB rates differ by race and geographic locale; the association between the vaginal microbiota and PTB also appears to differ, and race/ locale should be considered in evaluations of this association.

In our study, Lactobacillus-dominated communities had an inverse association with PTB among women swabbed before 12 weeks' but a direct association among those swabbed at or after 12 weeks' gestation. We observed effect modification on the multiplicative scale. These findings fit with observations in longitudinal studies-for example, Stout et al. found greater decreases in vaginal microbiome diversity over pregnancy in women with PTB than term birth [17] and Fettweis et al. found increases in L. crispatus abundance over the course of pregnancy among African American women who delivered preterm [18]. However, our analysis was post hoc, taking advantage of variation in timing of vaginal swab

Table 3

Results of logistic regression for preterm birth status stratified by gestational age at vaginal swab (odds ratios and $95 \% \mathrm{CI}$ ) in a sample of Peruvian women

\begin{tabular}{|c|c|c|c|c|c|c|}
\hline \multirow[t]{2}{*}{ Characteristic } & \multicolumn{3}{|c|}{ Sampled $<12$ wk' gestation } & \multicolumn{3}{|c|}{ Sampled $>12 w k-16 w^{\prime}$ gestation } \\
\hline & $\begin{array}{l}\text { Model } 1^{*} \text { OR }(95 \% \mathrm{CI})^{5} \\
15 \text { cases/54 controls }\end{array}$ & $\begin{array}{l}\text { Model } 2^{\dagger} \text { OR }(95 \% \mathrm{CI})^{\S} \\
15 \text { cases/53 controls }\end{array}$ & $\begin{array}{l}\text { Model } 3^{\ddagger} \text { OR }(95 \% \mathrm{CI})^{5} \\
15 \text { cases/53 controls }\end{array}$ & $\begin{array}{l}\text { Model } 1 * \text { OR }(95 \% \mathrm{CI})^{8} \\
10 \text { cases/45 controls }\end{array}$ & $\begin{array}{l}\text { Model } 2^{\dagger} \text { OR }(95 \% \mathrm{CI})^{8} \\
10 \text { cases/45 controls }\end{array}$ & $\begin{array}{l}\text { Model } 3^{\ddagger} \text { OR }(95 \% \mathrm{CI})^{8} \\
10 \text { cases } / 45 \text { controls }\end{array}$ \\
\hline $\begin{array}{l}\text { Lactobacillus ASV2 } \\
\text { dominated (reference } \\
\text { diverse community) }\end{array}$ & $0.49(0.12,1.78)$ & $0.49(0.12,1.82)$ & $0.49(0.11,1.81)$ & $5.33^{\text {ๆ }}(0.97,32.91)$ & $5.06^{6}(0.9,31.91)$ & $4.65^{\pi}(0.81,29.96)$ \\
\hline $\begin{array}{l}\text { L. iners dominated } \\
\text { (reference diverse } \\
\text { community) }\end{array}$ & $0.33(0.05,1.54)$ & $0.31(0.04,1.45)$ & $0.31(0.04,1.45)$ & $2.80(0.45,17.45)$ & $2.71(0.43,17.02)$ & $3.48(0.52,23.92)$ \\
\hline \multicolumn{7}{|c|}{$\begin{array}{l}* \text { Unadjusted. } \\
\text { Adjusted for parity status (one individual missing parity status; one individual missing gestational age at swab). } \\
\text { Adjusted for parity and Mestizo status (one individual missing parity status; one individual missing gestational age at swab). } \\
\S \text { Profile likelihood-based confidence intervals reported. } \\
\| \text { The second CSTs dominating organism-labeled ASV2-was identified as either L. acidophilus or L. crispatus by a BLAST search. }\end{array}$} \\
\hline
\end{tabular}


collection and we lacked repeated measures of the vaginal microbiome over pregnancy. Therefore, we cannot disentangle causal from noncausal mechanisms of the observed effect heterogeneity. It is possible that the women sampled before 12 weeks are not exchangeable with those sampled at or after 12 weeks' gestation. Although we controlled for important confounding factors, we cannot exclude the possibility of residual confounding by unmeasured variables such as administration of vaginal progesterone during pregnancy or placement of cervical cerclage.

Our study has other limitations that should also be considered. Our negative controls had very low biomass and were not sequenced. Thus, we cannot assess low-level contaminants. However, low-level contamination is unlikely to have severely affected our CSTs, which were characterized by typical, high-abundance vaginal organisms. In addition, we could not confidently assign a species level identification to the Lactobacillus sequence dominating our second community. Confidence intervals in the stratified analysis were large, due in part to the relatively small sample size-sample size may have impacted our power to detect differences. Future studies exploring the association between serial measures of vaginal microbiome across pregnancy and risk of PTB are warranted.

Our study has several strengths. First, our study was conducted in a well-characterized Peruvian cohort, and is one of only a handful of such studies in Latin America; the results highlight similarities and differences in pregnant vaginal microbiomes in different countries. Second, the determination of vaginal microbiomes collected during the first and early second trimester of gestation served to clarify the temporal relationship between changing microbiome community characteristics and subsequent risk of PTB. Third, our use of high-throughput 16S rRNA gene sequence-based methods for microbial profiling allowed for simultaneously investigating the entire microbial community.

In conclusion, in this cohort of Peruvian women, a Lactobacillusdominated CST did not appear protective for PTB. We found no association between microbial communities and PTB but did observe potential effect heterogeneity between vaginal communities and PTB by gestational age at microbiome assessment. Our findings may indicate the importance of considering race/ethnicity, geographic locale, and timing of vaginal sampling in studies of PTB and vaginal microbiomes.

\section{Acknowledgments}

This research was supported by an award from the Eunice Kennedy Shriver Institute of Child Health and Human Development, United States (R01-HD-059835).

\section{Supplementary data}

Supplementary data to this article can be found online at https://doi.org/10.1016/j.annepidem.2019.11.004.

\section{References}

[1] Blencowe H, Cousens S, Oestergaard MZ, Chou D, Moller A-B, Narwal R, et al. National, regional, and worldwide estimates of preterm birth rates in the year 2010 with time trends since 1990 for selected countries: a systematic analysis and implications. Lancet 2012;379(9832):2162-72.

[2] Romero R, Hassan SS, Gajer P, Tarca AL, Fadrosh DW, Bieda J, et al. The vaginal microbiota of pregnant women who subsequently have spontaneous preterm labor and delivery and those with a normal delivery at term. Microbiome 2014;2(1):18.

[3] Frey HA, Klebanoff MA. The epidemiology, etiology, and costs of preterm birth. Semin Fetal Neonatal Med 2016;21(2):68-73.
[4] Goldenberg RL, Culhane JF, Iams JD, Romero R. Epidemiology and causes of preterm birth. Lancet 2008:371(9606):75-84.

[5] for Disease Control C, Prevention. CDC. Preterm Birth. 2016. https://www.cdc gov/reproductivehealth/MaternalInfantHealth/PretermBirth.htm. [Accessed 16 October 2019].

[6] Lamont RF, Nhan-Chang C-L, Sobel JD, Workowski K, Conde-Agudelo A Romero R. Treatment of abnormal vaginal flora in early pregnancy with clindamycin for the prevention of spontaneous preterm birth: a systematic review and metaanalysis. Am J Obstet Gynecol 2011;205(3):177-90.

[7] Polettini J, Cobo T, Kacerovsky M, Vinturache AE, Laudanski P, Peelen MJCS et al. Biomarkers of spontaneous preterm birth: a systematic review of studies using multiplex analysis. J Perinat Med 2017;45(1):71-84.

[8] Brocklehurst P, Gordon A, Heatley E, Milan SJ. Antibiotics for treating bacterial vaginosis in pregnancy. Cochrane Database Syst Rev 2013;(1): CD000262.

[9] Foxman B, Wen A, Srinivasan U, Goldberg D, Marrs CF, Owen J, et al. Mycoplasma, bacterial vaginosis-associated bacteria BVAB3, race, and risk of preterm birth in a high-risk cohort. Am J Obstet Gynecol 2014;210(3): 226.e1-7.

[10] Mysorekar IU, Cao B. Microbiome in parturition and preterm birth. Semin Reprod Med 2014;32(1):50-5.

[11] Petricevic L, Domig KJ, Nierscher FJ, Sandhofer MJ, Fidesser M, Krondorfer I, et al. Characterisation of the vaginal Lactobacillus microbiota associated with preterm delivery. Sci Rep 2014;4:5136.

[12] Fox C, Eichelberger K. Maternal microbiome and pregnancy outcomes. Ferti Steril 2015;104(6):1358-63.

[13] Callahan BJ, DiGiulio DB, Goltsman DSA, Sun CL, Costello EK, Jeganathan P, et al. Replication and refinement of a vaginal microbial signature of preterm birth in two racially distinct cohorts of US women. Proc Natl Acad Sci U S A 2017;114(37):9966-71.

[14] DiGiulio DB, Callahan BJ, McMurdie PJ, Costello EK, Lyell DJ, Robaczewska A et al. Temporal and spatial variation of the human microbiota during pregnancy. Proc Natl Acad Sci U S A 2015;112(35):11060-5.

[15] Kindinger LM, Bennett PR, Lee YS, Marchesi JR, Smith A, Cacciatore S, et al. The interaction between vaginal microbiota, cervical length, and vaginal progesterone treatment for preterm birth risk. Microbiome 2017;5(1):6.

[16] Aagaard K, Riehle K, Ma J, Segata N, Mistretta T-A, Coarfa C, et al A Metagenomic Approach to Characterization of the Vaginal Microbiome Signature in Pregnancy. PLoS One 2012;7(6):e36466.

[17] Stout MJ, Zhou Y, Wylie KM, Tarr PI, Macones GA, Tuuli MG. Early pregnancy vaginal microbiome trends and preterm birth. Am J Obstet Gynecol 2017;217(3):356.e1-356.e18.

[18] Fettweis JM, Serrano MG, Brooks JP, Edwards DJ, Girerd PH, Parikh HI, et al The vaginal microbiome and preterm birth. Nat Med 2019;25(6):1012-21.

[19] Barrios YV, Sanchez SE, Nicolaidis C, Garcia PJ, Gelaye B, Zhong Q, et al. Childhood abuse and early menarche among peruvian women. J Adolesc Health 2015;56(2):197-202.

[20] The American College of Obstetricians and Gynecologists. Management of Preterm Labor: Practice Bulletin Number 171. 2016. https://www.acog.org/ Clinical-Guidance-and-Publications/Practice-Bulletins-List?IsMobileSet=false. Accessed 2019

[21] Savitz DA, Blackmore CA, Thorp JM. Epidemiologic characteristics of preterm delivery: Etiologic heterogeneity. Am J Obstet Gynecol 1991;164(2): 467-71.

[22] Williams M, Mittendorf R, Stubblefield PG, Lieberman E, Schoenbaum S, Monson RR. Cigarettes, coffee, and preterm premature rupture of the membranes. Am J Epidemiol 1992;135:895-903.

[23] Quast C, Pruesse E, Yilmaz P, Gerken J, Schweer T, Yarza P, et al. The SILVA ribosomal RNA gene database project: improved data processing and webbased tools. Nucleic Acids Res 2013:D590-6. https://doi.org/10.1093/nar/ gks1219.

[24] Morgan M. DirichletMultinomial: Dirichlet-Multinomial Mixture Model Machine Learning for Microbiome Data version 1.24.1 from Bioconductor 2017. https://rdrr.io/bioc/DirichletMultinomial/. [Accessed 5 February 2019].

[25] Fernandes AD, Macklaim JM, Linn TG, Reid G, Gloor GB. ANOVA-like differential expression (ALDEx) analysis for mixed population RNA-Seq. PLoS One 2013;8(7):e67019.

[26] Mathur MB, VanderWeele TJ. R function for additive interaction measures Epidemiology 2018;29(1):e5-6.

[27] VanderWeele T. Explanation in Causal Inference: Methods for Mediation and Interaction. New York: Oxford University Press, Incorporated; 2015. http:// ebookcentral.proquest.com/lib/umichigan/detail.action?docID=1920742. [Accessed 16 October 2019].

[28] Ravel J, Gajer P, Abdo Z, Schneider GM, Koenig SSK, McCulle SL, et al. Vaginal microbiome of reproductive-age women. Proc Natl Acad Sci U S A 2011;108(Suppl 1):4680-7.

[29] Zhou X, Brown CJ, Abdo Z, Davis CC, Hansmann MA, Joyce P, et al. Differences in the composition of vaginal microbial communities found in healthy Caucasian and black women. ISME J 2007:1(2):121-33.

[30] Hyman RW, Fukushima M, Jiang H, Fung E, Rand L, Johnson B, et al. Diversity of the vaginal microbiome correlates with preterm birth. Reprod Sc 2014;21(1):32-40. 